\title{
A STUDY OF A SPECIAL KIND OF $N$-FIXED POINT EQUATION SYSTEM AND APPLICATIONS
}

\author{
YONGFU SU, YINGLIN LUO, ADRIAN PETRUŞEL, AND JEN-CHIH YAO \\ Received 07 May, 2016
}

\begin{abstract}
The purpose of this paper is to consider a system of $N$-fixed point equations in metric spaces. The existence and uniqueness of solution and an iterative algorithm for approximating the solution are studied. This system of $N$-fixed point equations is an extension of the classical of fixed point equation $x=T x$. The results of this paper improve several important works recently published in the literature.
\end{abstract}

2010 Mathematics Subject Classification: 47H10; 54H25

Keywords: contraction mapping principle, complete metric spaces, multivariate fixed point, multiply metric function, system of $N$-fixed point operator equations, initial value problem

\section{INTRODUCTION}

Banach's contraction principle is one of the most powerful tools in applied nonlinear analysis. Weak contractions (also called $\phi$-contractions), as generalizations of Banach contraction mappings, have been studied by several authors. Let $T$ be a selfmap of a metric space $(X, d)$ and $\phi:[0,+\infty) \rightarrow[0,+\infty)$ be a function. We say that $T$ is a $\phi$-contraction if

$$
d(T x, T y) \leq \phi(d(x, y)), \quad \forall x, y \in X .
$$

In 1968, Browder [2] proved that if $\phi$ is non-decreasing and right continuous and $(X, d)$ is complete, then $T$ has a unique fixed point $x^{*} \in X$ and $\lim _{n \rightarrow \infty} T^{n} x_{0}=x^{*}$ for any given $x_{0} \in X$. Subsequently, this result was extended in 1969 by Boyd and Wong [1] by weakening the hypothesis on $\phi$, in the sense that it is sufficient to assume that $\phi$ is right upper semi-continuous and not necessarily monotone. For other results of this type see also [3]. For a comprehensive study of relations between several such contraction type conditions, see [7] and [8].

On the other hand, in 2015, Su and Yao [14] proved the following generalized contraction mapping principle.

For this work, the fourth author was partially supported by the Grant MOST 103-2923-E-039-001MY3. 
Theorem 1. Let $(X, d)$ be a complete metric space. Let $T: X \rightarrow X$ be a mapping such that

$$
\psi(d(T x, T y)) \leq \phi(d(x, y)), \forall x, y \in X,
$$

where $\psi, \phi:[0,+\infty) \rightarrow[0,+\infty)$ are two functions satisfying the conditions:

(1) $\psi(a) \leq \phi(b) \Rightarrow a \leq b$;

(2) $\left\{\begin{array}{l}\psi\left(a_{n}\right) \leq \phi\left(b_{n}\right) \\ a_{n} \rightarrow \varepsilon, b_{n} \rightarrow \varepsilon\end{array} \quad \Rightarrow \varepsilon=0\right.$.

Then, $T$ has a unique fixed point and, for any given $x_{0} \in X$, the iterative sequence $T^{n} x_{0}$ converges to this fixed point.

In particular, the study of the fixed points and coupled fixed points for weak contractions and generalized contractions was extended to partially ordered metric spaces in $[4-6,10-12,15]$. Among them, some results involve altering distance functions. Such functions were introduced by Khan et al. in [9], where some fixed point theorems are presented.

Recently, Y. Su, A. Petruşel and J. C. Yao [13] proved a multivariate contraction mapping principle in complete metric spaces.

The purpose of this paper is to consider a of system of $N$-fixed point equations in metric spaces. The existence and uniqueness of solution and the iterative algorithm of solution are studied. We notice that the system of $N$-fixed point equations is a generalized form of the fixed point equation $x=T x$. The results of this paper improve several important works published recently in the literature.

\section{A SYSTEM OF NONLINEAR EQUATIONS WITH CONTRACTION TYPE OPERATORS}

We will start the section with some concepts and results which are useful in our approach.

Definition 1. A multiply metric function $\triangle\left(a_{1}, a_{2}, \cdots, a_{N}\right)$ is a continuous $N$ variables non-negative real function with the domain

$$
\left\{\left(a_{1}, a_{2}, \cdots, a_{N}\right) \in \mathbb{R}^{N}: a_{i} \geq 0, i \in\{1,2,3, \cdots, N\}\right\}
$$

which satisfies the following conditions:

(1) $\triangle\left(a_{1}, a_{2}, \cdots, a_{N}\right)$ is non-decreasing for each variable $a_{i}, i \in\{1,2, \cdot \cdot N\}$,

(2) $\triangle\left(a_{1}+b_{1}, a_{2}+b_{2}, \cdots, a_{N}+b_{N}\right) \leq \triangle\left(a_{1}, a_{2}, \cdots, a_{N}\right)+\triangle\left(b_{1}, b_{2}, \cdots, b_{N}\right)$,

(3) $\triangle(a, a, \cdots, a)=a$,

(4) $\triangle\left(a_{1}, a_{2}, \cdots, a_{N}\right) \rightarrow 0 \Leftrightarrow a_{i} \rightarrow 0, i \in\{1,2,3, \cdots, N\}$,

(5) $\triangle\left(a_{1}, a_{2}, \cdots, a_{N}\right)=\triangle\left(a_{i_{1}}, a_{i_{2}}, \cdots, a_{i_{N}}\right)$,

for all $a_{i}, b_{i}, a \in \mathbb{R}, i \in\{1,2,3, \cdots, N\}$, where $a_{i_{1}}, a_{i_{2}}, \cdots, a_{i_{N}}$ is an arbitrary permutation of elements $a_{1}, a_{2}, \cdots, a_{N}$.

The following are some basic examples of multiply metric functions. 
Example 1. $\triangle_{1}\left(a_{1}, a_{2}, \cdots, a_{N}\right)=\frac{1}{N} \sum_{i=1}^{N} a_{i}$.

Example 2. $\triangle_{2}\left(a_{1}, a_{2}, \cdots, a_{N}\right)=\frac{1}{h} \sum_{i=1}^{N} q_{i} a_{i}$, where $q_{i} \in[0,1), i \in\{1, \cdots, N\}$

and $0<h:=\sum_{i=1}^{N} q_{i}<1$

Example 3. $\triangle_{3}\left(a_{1}, a_{2}, \cdots, a_{N}\right)=\sqrt{\frac{1}{N} \sum_{i=1}^{N} a_{i}^{2}}$.

Example 4. $\triangle_{4}\left(a_{1}, a_{2}, \cdots, a_{N}\right)=\max \left\{a_{1}, a_{2}, \cdots, a_{N}\right\}$.

An important concept is now presented.

Definition 2. Let $(X, d)$ be a metric space, $T: X^{N} \rightarrow X$ be a $N$-variables mapping, an element $p \in X$ is called a multivariate fixed point (or a fixed point of order $N$, see [13]) of $T$ if

$$
p=T(p, p, \cdots, p) .
$$

In what follows, we recall the following theorem which is a generalization of Banach's contraction principle. This theorem was proved by Y. Su, A. Petruşel and J. C. Yao in 2016, see [11].

Theorem 2. Let $(X, d)$ be a complete metric space and $T: X^{N} \rightarrow X$ be a $N$ variables mapping for which there exists $h \in(0,1)$ such that the following condition holds

$$
d(T x, T y) \leq h \triangle\left(d\left(x_{1}, y_{1}\right), d\left(x_{2}, y_{2}\right), \cdots, d\left(x_{N}, y_{N}\right)\right), \forall x, y \in X^{N},
$$

where $\triangle$ is a multiply metric function.

Then, $T$ has a unique multivariate fixed point $p \in X$ and, for any $p_{0} \in X^{N}$, the iterative sequence $\left\{p_{n}\right\} \subset X^{N}$ defined by

$$
\begin{aligned}
p_{1} & =\left(T p_{0}, T p_{0}, \cdots, T p_{0}\right) \\
p_{2} & =\left(T p_{1}, T p_{1}, \cdots, T p_{1}\right) \\
& \ldots \\
p_{n+1} & =\left(T p_{n}, T p_{n}, \cdots, T p_{n}\right)
\end{aligned}
$$

converges, in the multiply metric $\triangle$, to $(p, p, \cdots, p) \in X^{N}$ and the iterative sequence $\left\{T p_{n}\right\} \subset X$ converges, with respect to $d$, to $p \in X$.

In this article, we will extend Banach's contraction principle in another direction. 
Definition 3. Let $(X, d)$ be a metric space, $T: X^{N} \rightarrow X$ be a $N$-variables mapping, we consider the following $N$-variables system of equations:

$$
\left\{\begin{array}{c}
T\left(x_{1,1}, x_{1,2}, \cdots, x_{1, N}\right)=x_{1} \\
\cdots \\
T\left(x_{i, 1}, x_{i, 2}, \cdots, x_{i, N}\right)=x_{i} \\
\cdots \\
T\left(x_{N, 1}, x_{N, 2}, \cdots, x_{N, N}\right)=x_{N}
\end{array}\right.
$$

The system of equations (2.1) is said to be system of $N$-fixed point equations, where $x_{i, 1}, x_{i, 2}, \cdots, x_{i, N}, i=1,2, \cdots, N$ and $x_{1, j}, x_{2, j}, \cdots, x_{N, j}, j=1,2, \cdots, N$ are the permutations of elements $x_{1}, x_{2}, x_{3}, \cdots, x_{N}$.

Remark 1. It is easy to see that system (2.1) includes $\prod_{n=1}^{N} n$ ! systems of equations. For example, if $N=2$, then (2.1) includes $\prod_{n=1}^{2} n !=2 ! \cdot 1 !=2$ systems of coupled fixed point operator equations, i.e., the following systems

$$
\left\{\begin{array} { l } 
{ T ( x _ { 1 } , x _ { 2 } ) = x _ { 1 } } \\
{ T ( x _ { 2 } , x _ { 1 } ) = x _ { 2 } }
\end{array} \quad \left\{\begin{array}{l}
T\left(x_{1}, x_{2}\right)=x_{2} \\
T\left(x_{2}, x_{1}\right)=x_{1} .
\end{array}\right.\right.
$$

Example 5. Let $(X, d)$ be a metric space and $T: X^{N} \rightarrow X$. We consider the following systems of equations:

$$
\left\{\begin{array}{c}
T\left(x_{1}, x_{2}, \cdots, x_{N}\right)=x_{1}, \\
T\left(x_{2}, x_{3}, \cdots, x_{1}\right)=x_{2}, \\
\cdots \\
\cdots \\
T\left(x_{N}, x_{1}, \cdots, x_{N-1}\right)=x_{N},
\end{array}\right.
$$

and

$$
\left\{\begin{array}{l}
T\left(x_{1}, x_{2}, \cdots, x_{N}\right)=x_{N} \\
T\left(x_{2}, x_{3}, \cdots, x_{1}\right)=x_{1}, \\
\quad \cdots \\
\cdots \\
T\left(x_{N}, x_{1}, \cdots, x_{N-1}\right)=x_{N-1} .
\end{array}\right.
$$


The above systems of equations are also special forms of a system of $N$-fixed point equations. Moreover, the system (2.3) can be re-written as

$$
\left\{\begin{array}{l}
T\left(x_{2}, x_{3}, \cdots, x_{1}\right)=x_{1}, \\
T\left(x_{3}, x_{4}, \cdots, x_{2}\right)=x_{2}, \\
\quad \cdots \\
\quad \cdots \\
T\left(x_{N}, x_{1}, \cdots, x_{N-1}\right)=x_{N-1}, \\
T\left(x_{1}, x_{2}, \cdots, x_{N}\right)=x_{N},
\end{array}\right.
$$

In what follows, we prove our first main theorem, which generalizes Banach's contraction principle. We need first the following auxiliary notions and results.

Definition 4. Let $(X, d)$ be a complete metric space and define the following multiply metric $D$ given by

$$
D\left(\left(x_{1}, \cdots, x_{N}\right),\left(y_{1}, \cdots, y_{N}\right)\right)=\triangle\left(d\left(x_{1}, y_{1}\right), \cdots, d\left(x_{N}, y_{N}\right)\right)
$$

for all $\left(x_{1}, \cdots, x_{N}\right),\left(y_{1}, \cdots, y_{N}\right) \in X^{N}$.

Notice that the functional $D$ is a metric on $X^{N}$. Indeed, the following two conditions are obvious:

(i) $D\left(\left(x_{1}, \cdots, x_{N}\right),\left(y_{1}, \cdots, y_{N}\right)\right)=0 \Leftrightarrow\left(x_{1}, \cdots, x_{N}\right)=\left(y_{1}, \cdots, y_{N}\right)$;

(ii) $D\left(\left(y_{1}, \cdots, y_{N}\right)\right),\left(x_{1}, \cdots, x_{N}\right)=D\left(\left(x_{1}, \cdots, x_{N}\right),\left(y_{1}, \cdots, y_{N}\right)\right)$, for all $\left(x_{1}, \cdots, x_{N}\right),\left(y_{1}, \cdots, y_{N}\right) \in X^{N}$.

Next we prove the triangular inequality. For all

$$
\left(x_{1}, \cdots, x_{N}\right),\left(y_{1}, \cdots, y_{N}\right),\left(z_{1}, \cdots, z_{N}\right) \in X^{N},
$$

from the definition of $\triangle$, we have that

$$
\begin{aligned}
D\left(\left(x_{1}, \cdots, x_{N}\right),\left(y_{1}, \cdots\right.\right. & \left.\left., y_{N}\right)\right)=\triangle\left(d\left(x_{1}, y_{1}\right), \cdots, d\left(x_{N}, y_{N}\right)\right) \\
& \leq \triangle\left(d\left(x_{1}, z_{1}\right)+d\left(z_{1}, y_{1}\right), \cdots, d\left(x_{N}, z_{N}\right)+d\left(z_{N}, y_{N}\right)\right) \\
& \leq \triangle\left(d\left(x_{1}, z_{1}\right), \cdots, d\left(x_{N}, z_{N}\right)\right)+\triangle\left(d\left(z_{1}, y_{1}\right), \cdots, d\left(z_{N}, y_{N}\right)\right) \\
& =D\left(\left(x_{1}, \cdots, x_{N}\right),\left(z_{1}, \cdots, z_{N}\right)\right)+D\left(\left(z_{1}, \cdots, z_{N}\right),\left(y_{1}, \cdots, y_{N}\right)\right) .
\end{aligned}
$$

Moreover, if the metric space $(X, d)$ is complete, then we can prove that $\left(X^{N}, D\right)$ is a complete metric space. Indeed, let $\left\{p_{n}\right\} \subset X^{N}$ be a Cauchy sequence, then we have

$$
\lim _{n, m \rightarrow \infty} D\left(p_{n}, p_{m}\right)=\lim _{n, m \rightarrow \infty} \triangle\left(d\left(x_{1, n}, x_{1, m}\right), \cdots, d\left(x_{N, n}, x_{N, m}\right)\right)=0,
$$

where $p_{n}=\left(x_{1, n}, x_{2, n}, \cdots, x_{N, n}\right), p_{m}=\left(x_{1, m}, x_{2, m}, \cdots, x_{N, m}\right)$. From the definition of $\triangle$, we have that

$$
\lim _{n, m \rightarrow \infty} d\left(x_{i, n}, x_{i, m}\right)=0, \forall i \in\{1,2,3, \cdots, N\}
$$


Hence each $\left\{x_{i, n}\right\}(i \in\{1,2, \cdots, N\})$ is a Cauchy sequence. Since $(X, d)$ is a complete metric space, there exist $x_{1}, x_{2}, \cdots, x_{N} \in X$ such that, for all $i \in\{1,2, \cdots, N\}$ we have $\lim _{n \rightarrow \infty} d\left(x_{i, n}, x_{i}\right)=0$. Therefore $\lim _{n \rightarrow \infty} D\left(p_{n}, x\right)=0$, where $x=\left(x_{1}, x_{2}, \cdots, x_{N}\right) \in X^{N}$. Thus, the pair $\left(X^{N}, D\right)$ is a complete metric space.

Theorem 3. Let $(X, d)$ be a complete metric space and $T: X^{N} \rightarrow X$ be a $N$ variables mapping for which there exists $h \in(0,1)$ such that, for all $x=\left(x_{1}, \cdots, x_{N}\right), y=\left(y_{1}, \cdots, y_{N}\right) \in X^{N}$, the following condition is satisfied:

$$
d(T x, T y) \leq h \triangle\left(d\left(x_{1}, y_{1}\right), \cdots, d\left(x_{N}, y_{N}\right)\right),
$$

where $\triangle$ is a multiply metric function.

Then, the system of $N$-fixed point equations (2.1) has a unique solution $p=\left(p_{1}, \cdots, p_{N}\right)$ and for any $u_{0}=\left(x_{1}^{0}, \cdots, x_{N}^{0}\right) \in X^{N}$, the Picard iterative sequence $\left\{u_{n}\right\} \subset X^{N}$ defined by $u_{n}:=T_{*}^{n}\left(u_{0}\right)$ converges, with respect to the multiply metric $D$, to $p \in X^{N}$, where the operator $T_{*}: X^{N} \rightarrow X^{N}$ is defined by

$$
T_{*}:\left(x_{1}, x_{2}, \cdots, x_{N}\right) \mapsto\left(X_{1}, X_{2}, \cdots, X_{N}\right),
$$

where $X_{1}:=T\left(x_{1,1}, x_{1,2}, \cdots, x_{1, N}\right), \quad X_{2}:=T\left(x_{2,1}, x_{2,2}, \cdots, x_{2, N}\right), \cdots$, and $X_{N}:=$ $T\left(x_{N, 1}, x_{N, 2}, \cdots, x_{N, N}\right)$.

Proof. We consider on $X^{N}$ the define multiply metric $D$ given in Definition 4 . We prove that $T_{*}$ is a contraction from $\left(X^{N}, D\right)$ into itself. Observe that, for any $x=\left(x_{1}, x_{2}, \cdots, x_{N}\right), y=\left(y_{1}, y_{2}, \cdots, y_{N}\right) \in X^{N}$, we have that

$$
\begin{aligned}
D\left(T_{*} x, T_{*} y\right)=D & ( \\
& \left(T\left(x_{1,1}, x_{1,2}, \cdots, x_{1,2}, \cdots, y_{1, N}\right), \cdots, T\left(x_{N, 1}, x_{N, 2}, \cdots, x_{N, N}\right)\right), \\
=\triangle & \left(d\left(T\left(x_{1,1}, x_{1,2}, \cdots, x_{1, N}\right), T\left(y_{1,1}, y_{1,2}, \cdots, x_{1, N}\right)\right),\right. \\
& d\left(T\left(x_{2,1}, x_{2,2}, \cdots, x_{2, N}\right), T\left(y_{2,1}, y_{2,2}, \cdots, y_{2, N}\right)\right), \\
& \left.d\left(T\left(x_{N, 1}, x_{N, 2}, \cdots, x_{N, N}\right), T\left(y_{N, 1}, y_{N, 2}, \cdots, y_{N, N}\right)\right)\right) \\
\leq \triangle & \left(h \triangle\left(d\left(x_{1,1}, y_{1,1}\right), d\left(x_{1,2}, y_{1,2}\right), \cdots, d\left(x_{1, N}, y_{1, N}\right)\right),\right. \\
& h \triangle\left(d\left(x_{2,1}, y_{2,1}\right), d\left(x_{2,2}, y_{2,2}\right), \cdots, d\left(x_{2, N}, y_{2, N}\right)\right), \\
& \left.h \triangle\left(d\left(x_{3,1}, y_{3,1}\right), d\left(x_{3,2}, y_{3,2}\right), \cdots, d\left(x_{3, N}, y_{3, N}\right)\right)\right), \\
& \cdots \cdots, \\
& \left.h \triangle\left(d\left(x_{N, 1}, y_{N, 1}\right), d\left(x_{N, 2}, y_{N, 2}\right), \cdots, d\left(x_{N, N}, y_{N, N}\right)\right)\right)
\end{aligned}
$$

From the conditions (1), (5) of $\triangle$, we have

$$
\begin{aligned}
& \triangle\left(h \triangle\left(d\left(x_{1,1}, y_{1,1}\right), d\left(x_{1,2}, y_{1,2}\right), \cdots, d\left(x_{1, N}, y_{1, N}\right)\right),\right. \\
& h \triangle\left(d\left(x_{2,1}, y_{2,1}\right), d\left(x_{2,2}, y_{2,2}\right), \cdots, d\left(x_{2, N}, y_{2, N}\right)\right), \\
& \left.h \triangle\left(d\left(x_{3,1}, y_{3,1}\right), d\left(x_{3,2}, y_{3,2}\right), \cdots, d\left(x_{3, N}, y_{3, N}\right)\right)\right), \\
& \quad \cdots \cdots,
\end{aligned}
$$




$$
\begin{aligned}
& \left.h \triangle\left(d\left(x_{N, 1}, y_{N, 1}\right), \quad d\left(x_{N, 2}, y_{N, 2}\right), \cdots, d\left(x_{N, N}, y_{N, N}\right)\right)\right) \\
= & h \triangle\left(d\left(x_{1,1}, y_{1,1}\right), d\left(x_{1,2}, y_{1,2}\right), \cdots, d\left(x_{1, N}, y_{1, N}\right)\right) \\
= & h D\left(\left(x_{1}, x_{2}, \cdots, x_{N}\right),\left(y_{1}, y_{2}, \cdots, y_{N}\right)\right) \\
= & h D(x, y) .
\end{aligned}
$$

Hence

$$
D\left(T_{*} x, T_{*} y\right) \leq h D(x, y), \text { for all } x, y \in X^{N} .
$$

By Banach's contraction principle, we obtain that there exists a unique element $p=$ $\left(p_{1}, \cdots, p_{N}\right) \in X^{N}$ such that $p=T_{*} p$ and, for any element $u_{0} \in X^{N}$, the iterative sequence $u_{n}=T_{*}^{n}\left(u_{0}\right)$ converges, in the multiply metric $D$, to $p$. That is

$$
T_{*}\left(p_{1}, p_{2}, p_{3}, \cdots, p_{N}\right)=\left(p_{1}, p_{2}, p_{3}, \cdots, p_{N}\right) .
$$

From the definition of $T_{*}$, we have

$$
T_{*}\left(p_{1}, p_{2}, p_{3}, \cdots, p_{N}\right)=\left(P_{1}, P_{2}, P_{3}, \cdots, P_{N}\right),
$$

where

$$
\begin{aligned}
P_{1} & =T\left(p_{1,1}, p_{1,2}, \cdots, p_{1, N}\right), \\
P_{2} & =T\left(p_{2,1}, p_{2,2}, \cdots, p_{2, N}\right), \\
& \cdots \\
P_{N} & =T\left(p_{N, 1}, p_{N, 2}, \cdots, p_{N, N}\right) .
\end{aligned}
$$

Therefore, the vector $p=\left(p_{1}, p_{2}, \cdots, p_{N}\right) \in X^{N}$ is the unique solution of the system of $N$-fixed point equations (2.1). This completes the proof.

Notice that taking $N=1, \triangle(a)=a$ in Theorem 3, we obtain Banach's contraction mapping principle. Some other consequences of the above general result are the following corollaries.

Corollary 1. Let $(X, d)$ be a complete metric space and $T: X \times X \rightarrow X$ be a mapping for which there exists $h \in(0,1)$ such that

$$
d(T x, T y) \leq h \triangle\left(d\left(x_{1}, y_{1}\right), d\left(x_{2}, y_{2}\right)\right), \forall x=\left(x_{1}, x_{2}\right), y=\left(y_{1}, y_{2}\right) \in X \times X,
$$

where $\triangle$ is a multiply metric function.

Then, the system of coupled fixed point equations

$$
\left\{\begin{array}{l}
T\left(x_{1}, x_{2}\right)=x_{1}, \\
T\left(x_{2}, x_{1}\right)=x_{2} .
\end{array}\right.
$$

has a unique solution $p=\left(p_{1}, p_{2}\right)$ and for any $u_{0}=\left(x_{1}^{0}, x_{2}^{0}\right) \in X^{2}$, the Picard iterative sequence $\left\{u_{n}\right\} \subset X^{N}$ defined by $u_{n}=T_{*}^{n}\left(u_{0}\right)$ converges, in the multiply metric $D$, to $p \in X^{2}$, where the operator $T_{*}: X^{2} \rightarrow X^{2}$ is defined by

$$
T_{*}:\left(x_{1}, x_{2}\right) \mapsto\left(T\left(x_{1}, x_{2}\right), T\left(x_{2}, x_{1}\right)\right) .
$$


Notice that, in the above theorem, the detailed Picard iterative process is the following:

$$
\left\{\begin{array}{lc}
u_{0}= & \left(x_{1}^{0}, x_{2}^{0}\right), \\
u_{1}= & \left(T\left(x_{1}^{0}, x_{2}^{0}\right), T\left(x_{2}^{0}, x_{1}^{0}\right)\right), \\
u_{2}= & \left(T\left(T\left(x_{1}^{0}, x_{2}^{0}\right), T\left(x_{2}^{0}, x_{1}^{0}\right)\right), T\left(T\left(x_{2}^{0}, x_{1}^{0}\right), T\left(x_{1}^{0}, x_{2}^{0}\right)\right)\right), \\
\cdots &
\end{array}\right.
$$

Another consequence follows if we consider the multiply metric given in Example 3.

Corollary 2. Let $(X, d)$ be a complete metric space, $T: X^{N} \rightarrow X$ be a $N$-variables mapping for which there exists $h \in(0,1)$ such that

$$
d(T x, T y) \leq \frac{h}{N} \sum_{i=1}^{N} d\left(x_{i}, y_{i}\right), \forall x=\left(x_{1}, x_{2}, \cdots, x_{N}\right), y=\left(y_{1}, y_{2}, \cdots, y_{N}\right) \in X^{N} .
$$

Then, the system of $N$-fixed point equations (2.1) has a unique solution $p=$ $\left(p_{1}, p_{2}, \cdots, p_{N}\right)$ and, for any $u_{0}=\left(x_{1}^{0}, x_{2}^{0}, \cdots, x_{N}^{0}\right) \in X^{N}$, the Picard iterative sequence $\left\{u_{n}\right\} \subset X^{N}$ defined by $u_{n}=T_{*}^{n}\left(u_{0}\right)$ converges, in the multiply metric $D$, to $p \in X^{N}$, where the operator $T_{*}: X^{N} \rightarrow X^{N}$ is defined by

$$
T_{*}:\left(x_{1}, x_{2}, \cdots, x_{N}\right) \mapsto\left(X_{1}, X_{2}, \cdots, X_{N}\right),
$$

where

$$
\begin{aligned}
& X_{1}=T\left(x_{1,1}, x_{1,2}, x_{1,3}, \cdots, x_{1, N}\right), \\
& X_{2}=T\left(x_{2,1}, x_{2,2}, x_{2,3}, \cdots, x_{2, N}\right), \\
& \cdots, \\
& X_{N}=T\left(x_{N, 1}, x_{N, 2}, x_{N, 3}, \cdots, x_{N, N}\right) .
\end{aligned}
$$

Some other corollaries, given for different other multiply metrics, can be given. For example, we have the following two results.

Corollary 3. Let $(X, d)$ be a complete metric space and $T: X^{N} \rightarrow X$ be a $N$ variables mapping for which there exists $h \in(0,1)$ such that the following condition holds

$$
d(T x, T y) \leq h \sqrt{\frac{1}{N} \sum_{i=1}^{N} d\left(x_{i}, y_{i}\right)^{2}}, \forall x=\left(x_{1}, x_{2}, \cdots, x_{N}\right), y=\left(y_{1}, y_{2}, \cdots y_{N}\right) \in X^{N} .
$$

Then, the system of $N$-fixed point equations (2.1) has a unique solution $p=$ $\left(p_{1}, p_{2}, p_{3}, \cdots, p_{N}\right)$ and for any $u_{0}=\left(x_{1}^{0}, x_{2}^{0}, \cdots, x_{N}^{0}\right) \in X^{N}$, the Picard iterative sequence $\left\{u_{n}\right\} \subset X^{N}$ defined by $u_{n}=T_{*}^{n}\left(u_{0}\right)$ converges, in the multiply metric $D$, to $p \in X^{N}$. The operator $T_{*}: X^{N} \rightarrow X^{N}$ is defined by

$$
T_{*}:\left(x_{1}, x_{2}, \cdots, x_{N}\right) \mapsto\left(X_{1}, X_{2}, \cdots, X_{N}\right),
$$


where

$$
\begin{aligned}
& X_{1}=T\left(x_{1,1}, x_{1,2}, x_{1,3}, \cdots, x_{1, N}\right), \\
& X_{2}=T\left(x_{2,1}, x_{2,2}, x_{2,3}, \cdots, x_{2, N}\right), \\
& \cdots, \\
& X_{N}=T\left(x_{N, 1}, x_{N, 2}, x_{N, 3}, \cdots, x_{N, N}\right) .
\end{aligned}
$$

Corollary 4. Let $(X, d)$ be a complete metric space and $T: X^{N} \rightarrow X$ be a $N$ variables mapping for which there exists $h \in(0,1)$ such that, for all $x=\left(x_{1}, x_{2}, \cdot\right.$. $\left.\cdot, x_{N}\right), y=\left(y_{1}, y_{2}, \cdot y_{N}\right) \in X^{N}$, the following condition holds

$$
d(T x, T y) \leq h \max \left\{d\left(x_{1}, y_{1}\right), \cdots, d\left(x_{N}, y_{N}\right)\right\} .
$$

Then, the system of $N$-fixed point equations (2.1) has a unique solution $p=$ $\left(p_{1}, p_{2}, \cdots, p_{N}\right)$ and, for any $u_{0}=\left(x_{1}^{0}, x_{2}^{0}, \cdots, x_{N}^{0}\right) \in X^{N}$, the Picard iterative sequence $\left\{u_{n}\right\} \subset X^{N}$ defined by $u_{n}=T_{*}^{n}\left(u_{0}\right)$ converges, in the multiply metric $D$, to $p \in X^{N}$, where the operator $T_{*}: X^{N} \rightarrow X^{N}$ is defined by

$$
T_{*}:\left(x_{1}, x_{2}, \cdots, x_{N}\right) \mapsto\left(X_{1}, X_{2}, \cdots, X_{N}\right),
$$

where

$$
\begin{aligned}
& X_{1}=T\left(x_{1,1}, x_{1,2}, x_{1,3}, \cdots, x_{1, N}\right), \\
& X_{2}=T\left(x_{2,1}, x_{2,2}, x_{2,3}, \cdots, x_{2, N}\right), \\
& \cdots, \\
& X_{N}=T\left(x_{N, 1}, x_{N, 2}, x_{N, 3}, \cdots, x_{N, N}\right) .
\end{aligned}
$$

In particular, for the systems (2.2) and (2.3) we get the following results.

Corollary 5. Let $(X, d)$ be a complete metric space, $T: X^{N} \rightarrow X$ be a $N$-variables mapping for which there exists $h \in(0,1)$ such that, for all $x=\left(x_{1}, \cdots, x_{N}\right), y=$ $\left(y_{1}, \cdots, y_{N}\right) \in X^{N}$, the following condition holds

$$
d(T x, T y) \leq h \triangle\left(d\left(x_{1}, y_{1}\right), \cdots, d\left(x_{N}, y_{N}\right)\right),
$$

where $\triangle$ is a multiply metric function.

Then, the system of $N$-fixed point equations (2.2) has a unique solution $p=$ $\left(p_{1}, p_{2}, \cdots, p_{N}\right)$ and for any $u_{0}=\left(x_{1}^{0}, x_{2}^{0}, \cdots, x_{N}^{0}\right) \in X^{N}$, the Picard iterative sequence $\left\{u_{n}\right\} \subset X^{N}$ defined by $u_{n}=T_{*}^{n}\left(u_{0}\right)$ converges, in the multiply metric $D$, to $p \in X^{N}$, where the operator $T_{*}: X^{N} \rightarrow X^{N}$ is defined by

$$
T_{*}:\left(x_{1}, x_{2}, \cdots, x_{N}\right) \mapsto\left(X_{1}, X_{2}, \cdots, X_{N}\right),
$$

where

$$
\begin{aligned}
& X_{1}=T\left(x_{1}, x_{2}, x_{3}, \cdots, x_{N}\right), \\
& X_{2}=T\left(x_{2}, x_{3}, x_{4}, \cdots, x_{1}\right), \\
& \cdots
\end{aligned}
$$




$$
X_{N}=T\left(x_{N}, x_{1}, x_{2}, \cdots, x_{N-1}\right) .
$$

Corollary 6. Let $(X, d)$ be a complete metric space, $T: X^{N} \rightarrow X$ be a $N$-variables mapping for which there exists $h \in(0,1)$ such that, for all $x=\left(x_{1}, x_{2}, \cdots, x_{N}\right), y=$ $\left(y_{1}, y_{2}, \cdots, y_{N}\right) \in X^{N}$, the following condition holds

$$
d(T x, T y) \leq h \triangle\left(d\left(x_{1}, y_{1}\right), \cdots, d\left(x_{N}, y_{N}\right)\right),
$$

where $\triangle$ is a multiply metric function.

Then, the system of $N$-fixed point equations (2.3) has a unique solution $p=$ $\left(p_{1}, p_{2}, \cdots, p_{N}\right)$ and, for any $u_{0}=\left(x_{1}^{0}, x_{2}^{0}, \cdots, x_{N}^{0}\right) \in X^{N}$, the iterative sequence $\left\{u_{n}\right\} \subset$ $X^{N}$ defined by $u_{n}=T_{*}^{n}\left(u_{0}\right)$ converges, in the multiply metric $D$, to $p \in X^{N}$, where the operator $T_{*}: X^{N} \rightarrow X^{N}$ is defined by

$$
T_{*}:\left(x_{1}, x_{2}, \cdots, x_{N}\right) \mapsto\left(X_{1}, X_{2}, \cdots, X_{N}\right),
$$

where

$$
\begin{aligned}
& X_{1}=T\left(x_{2}, x_{3}, x_{4}, \cdots, x_{1}\right), \\
& X_{2}=T\left(x_{3}, x_{4}, x_{5}, \cdots, x_{2}\right), \\
& \cdots \\
& X_{N}=T\left(x_{1}, x_{2}, x_{3}, \cdots, x_{N}\right) .
\end{aligned}
$$

\section{AN APPLICATION TO A SYSTEM OF FIRST ORDER DIFFERENTIAL EQUATIONS}

We will give now an application of the above results to an initial value problem related to a system of first order differential equations of the following form:

$$
\left\{\begin{array}{c}
\frac{d x_{1}}{d t}=f\left(x_{1,1}(t), x_{1,2}(t), \cdots, x_{1, N}(t), t\right), \\
\quad \cdots \\
\frac{d x_{i}}{d t}=f\left(x_{i, 1}(t), x_{i, 2}(t), \cdots, x_{i, N}(t), t\right), \\
\quad \cdots \\
\frac{d x_{N}}{d t}=f\left(x_{N, 1}(t), x_{N, 2}(t), \cdots, x_{N, N}(t), t\right), \\
x_{i}\left(t_{0}\right)=x^{0}, \quad i=1,2,3, \cdots, N .
\end{array}\right.
$$

where $t_{0} \in \mathbb{R}$. We denote $I:=\left[t_{0}-\delta, t_{0}+\delta\right]$ (where $\delta>0$ is a given real number) and consider $f: \mathbb{R}^{N} \times I \rightarrow \mathbb{R}$ be a continuous $(N+1)$-variables function satisfying the following Lipschitz type condition

$$
\left|f\left(x_{1}, x_{2}, \cdots, x_{N}, t\right)-f\left(y_{1}, y_{2}, \cdots, y_{N}, t\right)\right| \leq k(t) \sum_{i=1}^{N}\left|x_{i}-y_{i}\right|,
$$


with $k \in L^{1}\left(I, \mathbb{R}_{+}\right)$. We will consider first the following equivalent system of integral equations

$$
\left\{\begin{array}{c}
x_{1}(t)=\int_{t_{0}}^{t} f\left(x_{1,1}(\tau), x_{1,2}(\tau), \cdots, x_{1, N}(\tau), \tau\right) d \tau+x^{0}, \\
\cdots \\
x_{i}(t)=\int_{t_{0}}^{t} f\left(x_{i, 1}(\tau), x_{i, 2}(\tau), \cdots, x_{i, N}(\tau), \tau\right) d \tau+x^{0} \\
\cdots \\
x_{N}(t)=\int_{t_{0}}^{t} f\left(x_{N, 1}(\tau), x_{N, 2}(\tau), \cdots, x_{N, N}(\tau), \tau\right) d \tau+x^{0}
\end{array}\right.
$$

Let $X:=C\left[t_{0}-\delta, t_{0}+\delta\right]$ be the linear space of continuous real functions defined on closed interval $I:=\left[t_{0}-\delta, t_{0}+\delta\right]$. We introduce on $X$ a Bielecki type metric, by the relation

$$
d_{B}(x, y):=\max _{t_{0}-\delta \leq t \leq t_{0}+\delta}|x(t)-y(t)| e^{-L K(t)},
$$

where $K(t):=\int_{t_{0}}^{t} k(s) d s$ and $L$ is a constant greater than $N$.

Let $T: X \times X \times \cdots \times X \rightarrow X$ be a mapping defined by

$$
T x(t):=\int_{t_{0}}^{t} f\left(x_{1}(\tau), x_{2}(\tau), \cdots, x_{N}(\tau), \tau\right) d \tau+x^{0} .
$$

For any $x=\left(x_{1}, x_{2}, \cdots, x_{N}\right), y=\left(y_{1}, y_{2}, \cdots, y_{N}\right) \in X^{N}$ and $t \in I$ we have that

$$
\begin{aligned}
|T x(t)-T y(t)| & \leq\left|\int_{t_{0}}^{t}\right| f(x(\tau), \tau)-f(y(\tau), \tau)|d \tau| \\
& \leq\left|\int_{t_{0}}^{t} \sum_{i=1}^{N} k(\tau)\right| x_{i}(\tau)-y_{i}(\tau) d \tau \mid \\
& \leq\left|\int_{t_{0}}^{t}\right| \sum_{i=1}^{N} \max _{\tau \in I}\left[\left|x_{i}(\tau)-y_{i}(\tau)\right| e^{-L K(\tau)}\right] k(\tau) e^{L K(\tau)} d \tau \mid \\
& =N\left|\int_{t_{0}}^{t}\left(\frac{1}{N} \sum_{i=1}^{N} d_{B}\left(x_{i}, y_{i}\right)\right) k(\tau) e^{L K(\tau)} d \tau\right| \\
& =N \triangle_{1}\left(d_{B}\left(x_{1}, y_{1}\right), \cdots, d_{B}\left(x_{N}, y_{N}\right)\right)\left|\int_{t_{0}}^{t} k(\tau) e^{L K(\tau)} d \tau\right| \\
& \leq \frac{N}{L} \cdot \triangle_{1}\left(d_{B}\left(x_{1}, y_{1}\right), \cdots, d_{B}\left(x_{N}, y_{N}\right)\right) e^{L K(t)} .
\end{aligned}
$$

Thus,

$$
|T x(t)-T y(t)| e^{-L K(t)} \leq \frac{N}{L} \cdot \triangle_{1}\left(d_{B}\left(x_{1}, y_{1}\right), \cdots, d_{B}\left(x_{N}, y_{N}\right)\right), \text { for all } t \in I .
$$

Hence we get that

$$
d_{B}(T x, T y) \leq \frac{N}{L} \cdot \triangle_{1}\left(d_{B}\left(x_{1}, y_{1}\right), \cdots, d_{B}\left(x_{N}, y_{N}\right)\right), \text { for all } x, y \in X .
$$


Since $h:=\frac{N}{L}<1$, we conclude, by using Theorem 3, that the system of integral equations (3.2) has a unique solution

$$
x^{*}=\left(x_{1}^{*}(t), x_{2}^{*}(t), x_{3}^{*}(t), \cdots, x_{N}^{*}(t)\right) \in\left(C\left[t_{0}-\delta, t_{0}+\delta\right]\right)^{N} .
$$

Since the system (3.2) is equivalent to the system (3.1), by our approach, the existence and uniqueness result for (3.1) follows.

On the other hand, by Theorem 3, we also know that, for any initial value

$$
u_{0}=\left(x_{0,1}, x_{0,2}, x_{0,3}, \cdots, x_{0, N}\right) \in\left(C\left[t_{0}-\delta, t_{0}+\delta\right]\right)^{N},
$$

the iterative sequence $\left\{u_{n}\right\} \subset\left(C\left[t_{0}-\delta, t_{0}+\delta\right]\right)^{N}$ defined by $u_{n}=T_{*}^{n}\left(u_{0}\right)$ converges, in the multiply metric $D$, to the unique solution of the initial value problem (3.1) (i.e., $\left.x^{*} \in\left(C\left[t_{0}-\delta, t_{0}+\delta\right]\right)^{N}\right)$, where

$$
T_{*}:\left(C\left[t_{0}-\delta, t_{0}+\delta\right]\right)^{N} \rightarrow\left(C\left[t_{0}-\delta, t_{0}+\delta\right]\right)^{N}
$$

is defined by

$$
T_{*}:\left(x_{1}(t), x_{2}(t), x_{3}(t), \cdots, x_{N}(t)\right) \mapsto\left(X_{1}(t), X_{2}(t), X_{3}(t), \cdots, X_{N}(t)\right),
$$

with

$$
\left\{\begin{array}{c}
X_{1}(t)=\int_{t_{0}}^{t} f\left(x_{1,1}(\tau), x_{1,2}(\tau), \cdots, x_{1, N}(\tau), \tau\right) d \tau+x^{0}, \\
\cdots \\
X_{i}(t)=\int_{t_{0}}^{t} f\left(x_{i, 1}(\tau), x_{i, 2}(\tau), \cdots, x_{i, N}(\tau), \tau\right) d \tau+x^{0}, \\
\cdots \\
X_{N}(t)=\int_{t_{0}}^{t} f\left(x_{N, 1}(\tau), x_{N, 2}(\tau), \cdots, x_{N, N}(\tau), \tau\right) d \tau+x^{0},
\end{array}\right.
$$

and the multiply metric $D$ used here (see Example 1 ) is defined by

$$
D\left(\left(x_{1}, x_{2}, \cdots, x_{N}\right),\left(y_{1}, y_{2}, \cdots, y_{N}\right)\right)=\triangle_{1}\left(d_{B}\left(x_{1}, y_{1}\right), d_{B}\left(x_{2}, y_{2}\right), \cdots, d_{B}\left(x_{N}, y_{N}\right)\right),
$$

for all $\left(x_{1}, x_{2}, \cdots, x_{N}\right),\left(y_{1}, y_{2}, \cdots, y_{N}\right) \in\left(C\left[t_{0}-\delta, t_{0}+\delta\right]\right)^{N}$.

\section{REFERENCES}

[1] D. Boyd and J. Wong, "On nonlinear contractions," Proc. Amer. Math. Soc., vol. 20, pp. 458-464, 1969, doi: 10.1090/S0002-9939-1969-0239559-9.

[2] F. Browder, "On the convergence of successive approximations for nonlinear functional equations," Indag. Math., vol. 30, no. 1, pp. 27-35, 1968, doi: 10.1016/S1385-7258(68)50004-0.

[3] M. Geraghty, "On contractive mappings," Proc. Amer. Math. Soc., vol. 40, pp. 604-608, 1973, doi: 10.1090/S0002-9939-1973-0334176-5.

[4] T. Gnana Bhaskar and V. Lakshmikantham, "Fixed point theorems in partially ordered metric spaces and applications," Nonlinear Anal., vol. 65, no. 7, pp. 1379-1393, 2006, doi: 10.1016/j.na.2005.10.017.

[5] J. Harjani and K. Sadarangni, "Fixed point theorems for weakly contraction mappings in partially ordered sets," Nonlinear Anal., vol. 71, pp. 3403-3410, 2009, doi: 10.1016/j.na.2009.01.240.

[6] J. Harjani and K. Sadarangni, "Generalized contractions in partially ordered metric spaces and applications to ordinary differential equations," Nonlinear Anal., vol. 72, pp. 1188-1197, 2010, doi: 10.1016/j.na.2009.08.003. 
[7] J. Jachymski, "Equivalence of some contractivity properties over metrical structures," Proc. Amer. Math. Soc., vol. 125, pp. 2327-2335, 1997, doi: 10.1090/S0002-9939-97-03853-7.

[8] J. Jachymski and I. Jozwik, "Nonlinear contractive conditions: a comparison and related problems," in Fixed Point Theory and its Applications, ser. Polish Acad. Sci., Banach Center Publ., J. Jachymski and S. Reich, Eds., vol. 77, doi: 10.4064/bc77-0-10. Warsaw: PWN Wrasaw, 2007, pp. 123-146.

[9] M. Khan, M. Swaleh, and S. Sessa, "Fixed point theorems by altering distances between the points," Bull. Aust. Math. Soc., vol. 30, no. 1, pp. 1-9, 1984, doi: 10.1017/S0004972700001659.

[10] V. Lakshmikantham and L. Ciric, "Coupled fixed point theorems for nonlinear contractions in partially ordered metric spaces," Nonlinear Anal., vol. 70, no. 12, pp. 4341-4349, 2009, doi: 10.1016/j.na.2008.09.020.

[11] J. Nieto and R. Rodriguez-López, "Existence and uniqueness of fixed point in partially ordered sets and applications to ordinary differential equations," Acta Math. Sinica, vol. 23, no. 11, pp. 2205-2212, 2007, doi: 10.1007/s10114-005-0769-0.

[12] A. Petrusel, G. Petrusel, B. Samet, and J. Yao, "Coupled fixed point theorems for symmetric contractions in b-metric spaces with applications to operator equation systems," Fixed Point Theory, vol. 17, pp. 457-476, 2016.

[13] Y. Su, A. Petrusel, and J.-C. Yao, "Multivariate fixed point theorems for contractions and nonexpansive mappings with applications," Fixed Point Theory Appl., vol. 2016:19, pp. 1-19, 2016, doi: 10.1186/s13663-015-0493-0.

[14] Y. Su and J.-C. Yao, "Further generalized contraction mapping principle and best proximity theorem in metric spaces," Fixed Point Theory Appl., vol. 2015:120, pp. 1-13, 2015, doi: 10.1186/s13663-015-0373-7.

[15] F. Yan, Y. Su, and Q. Feng, "A new contraction mapping principle in partially ordered metric spaces and applications to ordinary differential equations," Fixed Point Theory Appl., vol. 2012:152, pp. 1-13, 2012, doi: 10.1186/1687-1812-2012-152.

Authors' addresses

Yongfu Su

Department of Mathematics, Tianjin Polytechnic University, Tianjin 300387, China

E-mail address: t jsuyongfue163.com

Yinglin Luo

Department of Mathematics, Tianjin Polytechnic University, Tianjin 300387, China

E-mail address: luoyinglink@163.com

Adrian Petruşel

Department of Mathematics, Babeş-Bolyai University Cluj-Napoca,, Kogălniceanu Str. No. 1, 400084, Cluj-Napoca, Romania and Academy of Romanian Scientists, Bucharest, Romania

E-mail address: petrusel@math.ubbcluj.ro

\section{Jen-Chih Yao}

Research Center for Interneural Computing, China Medical University Hospital, China Medical University, Taichung, Taiwan and Department of Applied Mathematics, National Sun Yat-sen University, Kaohsiung, Taiwan

E-mail address: yaojcemail.cmu.edu.tw 